\title{
Introducing a Novel Algorithm for Energy Consumption Management for M2M Network Expandability through Topology Control within Nodes
}

\author{
Mohammad Amini ${ }^{1}$, Akbar Majidi ${ }^{2 *}$ \\ ${ }^{I}$ Department of Computer Engineering and Information Technology of Amirkabir University of Technology \\ (Tehran Polytechnic), Tehran, Iran. \\ ${ }^{2}$ Networks Laboratory and Advance Programming (NLAP), www.NS-3.org Germi, Iran.
}

\begin{abstract}
The wireless sensor networks are most important developers of machine-to-machine communication (M2M) networks. In each node of sensor, transmission power is the most important energy consumption factor, which is considered as the most important challenge for expansibility of M2M network. One strategy to cope with this problem is topology control algorithm. This algorithm consists of two topology design and topology maintenance phases. The focus of most works conducted in this field is on design topography. However, topology maintenance should be also considered during the topology control design. To prolong network life through the energy consumption management in the nodes, in this paper an algorithm is proposed which considers both topology design and maintenance. The simulation results obtained using NS-2 simulators prove innovation and higher performance of the proposed model.
\end{abstract}

Index Terms: Machine-to-Machine communication, Energy efficiency, Topology control.

(C) 2014 Published by MECS Publisher. Selection and/or peer review under responsibility of the Research Association of Modern Education and Computer Science

\section{Introduction}

Wireless sensor networks are utilized in different M2M networks such as intelligent networks, healthcare monitoring, intelligent light control, house automation, and supervision systems. A strategy to deal with the challenge of optimum source consumption is topology control algorithm, which is an effective method for enhancing energy efficiency and prolonging network life. Topology control of this algorithm is performed as a repeated process consisting of two topology design and topology maintenance steps. The main impetus behind this topology is to develop an expansible topology through the source consumption management; in the second

* Corresponding author.

E-mail address: m.amini1@aut.ac.ir, majidi86@gmail.com 
part of this paper, the related works are reviews; third part introduces the proposed algorithm, which consist of topology design and topology maintenance; section 4 presents simulation results; finally, in section 5 conclusion of the conducted work is presented.

\section{Related works}

M2M technology supports wireless communications between two machines. This emerging technology has many applications for the potential new generation applications for improving network resource consumption and reducing the operational costs of the network. In Refs. [1,7] discusses shortest path selection routing and different types of communication technologies using the applied intelligent network program for M2M communications [4], as well as the challenges of M2M networks for a home energy management system within an intelligent network, and adaptive portioning using the connectivity-based portioning approach (CPA) for node timing and topology control in sensor networks. In addition, Sethu and Gerety studied topology control steps for reducing the energy consumption by keeping the connections in the non-homogenous sensor networks independent from sensor location. They performed their topology control for non-homogenous path error propagation [6]. In Ref. [7] a topology control algorithm is proposed in the form of backbone structure. Through this efficient algorithm, in the topology design phase of the proposed algorithm the transmitted messages for making an efficient algorithm are reduced; so that the proposed algorithm is considered as an efficient method. The authors of this work tried to improve topology control phase [5]. In Ref. [6] the topology control algorithm of Smart Boundary Yao Gabriel Graph (SBYaoGG) is introduced. The main applications of this algorithm are for control of consumed energy and reducing the mediate in wireless network sensors [9]. In Refs. $[9,10]$ a hierarchical routing algorithm was proposed for multiple node sink, as well as a procedure for collecting the general data [9]. Moreover, in Ref. [10] a topology was designed which is able to decrease the number of coordinators without any change in network links. This reduction in number of coordinates reduces the average work cycle and, consequently, prolonged life for energy consumption [10].

\section{Proposed algorithm}

As previously mentioned, transmission power is the most important factor for energy consumption in every sensor node, which is considered among the expansibility of M2M network. By having an algorithm for control of consumed energy of the network, it is possible to prolong network life, and reduce its energy consumption. Designing such algorithm would consequently result in an expansible network. Therefore, our goal is realized if each sensor node can adjust its topology for energy save via adjusting the transmission power by selecting the parent node which is directly link to. Here, parent node is called to a node that necessarily has a child and it can be a child of another parent or linked to sink node.

Once the topology phase is created, the topology maintenance phase initiates in each sensor node. The nodes of M2M network can be suddenly displaced or power on and off. This functions result in categorization of these nodes into: 1 . Nodes with limited supply source which are constantly on; 2 . Nodes with limited supply source which are intermittently on and off; 3 . Nodes with unlimited supply source which are constantly on; and 4 nodes with unlimited supply source which are intermittently on and off. Considering these categories, the parent of each sensor node is required to be adjusted in its appropriate time. Therefore, the maintenance phase monitors the topology situation and creates topology design phase, if needed. This cycle is repeated for a considerable share of network life until the energy is completely depleted. 


\subsection{Proposed network model}

The home-based M2M network consists of one external network and several subnets. The external network is a central home gat for management of the entire network, collecting the data from entire network, and connection of home network to the outside world (internet). Fig 1. Illustrates this structure.

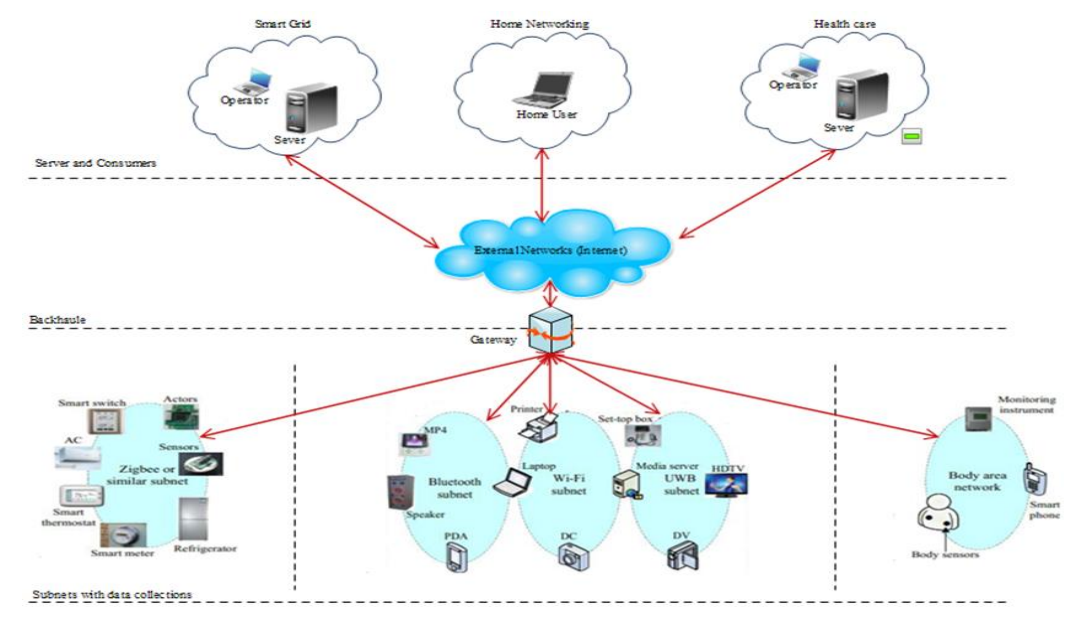

Fig. 1. Subnets of home-based M2M network.

Each subnet works in self-organization mode and is designed for a specific application. Subnets are linked together based on IEEE-802.15.4 protocol [11] [12]. Based on the mentioned points, now it is assumed that $\mathrm{N}$ equal sensors are randomly distributed in a given environment, where grid graph is presented as $G(R, E)$ in which $\mathrm{R}$ and $\mathrm{E}$ are a set of nodes and communication links, respectively. For instance, the distance between two sensor nodes $\mathrm{z}$ and $\mathrm{r}$ is represented as $\mathrm{d}(\mathrm{r}, \mathrm{z})$. Each sensor node can regulate its power to communicate with other nodes. To obtain the relationship and computation of transmission power $\rho t x$ (z), it must be noted that several parameters affect transmission power. One of these parameters is distance and another is threshold level. Moreover, it is known that transmission power have a direct exponential relationship with distance, which is named as $\mathrm{x}$ in the following equation which is indeed path loss factor. This exponential function varies in the range of 2 to 5 , which means that to compute transmission power, the distance between sensor nodes; $\mathrm{z}$ and $\mathrm{r}$ must have a 2 to 5 exponent and then be multiplied to the threshold level.

Transmission power of node $r$ to node $\mathrm{z}=$ threshold power $\times d(r, z)^{x}$

To build local graph, each sensor node can send and deliver Hello messages with maximum power for finding neighbor nodes, $\mathrm{N}(\mathrm{z})$. Based on $\mathrm{Eq} 1 ., \rho_{\min }(\mathrm{r}, \mathrm{z})$ presents minimum power needed for sending one packed from $\mathrm{r}$ to $\mathrm{z}$, Therefore, according to $\mathrm{N}(\mathrm{z})$ and $\rho \mathrm{m}_{\min }(\mathrm{r}, \mathrm{z})$, node $\mathrm{z}$ can have a sub-local graph. Here, $\rho_{\min }$ indicates the remaining energy in node $z$. Transmission power lever, $\rho_{\mathrm{pi}}(\mathrm{z})$ can be maximum or in the range between minimum to maximum in the entire network. Connection tree topology in the network is made of different nodes and optimum transmission power level. By analyzing the characteristics of the sensors, two classifications are possible in terms of the energy considerations: 1. If node $\mathrm{z}$ have unlimited supply source, transmission power level would be "decrease order" type; 2 . If the supply source is limited (limited battery), transmission power level is "increaser order" type. In the short distances, transmission power costs are reduced, 
while, the system life is prolonged. To obtain power transmission levels, node $\mathrm{z}$ finds node $\mathrm{r}$ with maximum remaining energy and shortest path. The short path indicates number of "hops", where Hop Count (z) is greater than or equal to Hop Count(r) in this algorithm.

\subsection{Topology design for the proposed model}

Topology control creates a compatible network for improving energy storage and a prolonged network life through the network adjustment. In the topology design step, the low energy consumption topological feature contributes formation of dynamic topological structures for keeping connections in the network. Now, to develop a tree topology, each sensor creates a local graph (containing all neighbouring nodes with maximum transmission power) and selects one of neighbouring nodes as parent node, $\mathrm{P}(\mathrm{z})$. Selection of parent node is based on the maximum remaining energy. Therefore, a tree-based backbone can be developed for data transmission. Now, based on different types of mentioned nodes for M2M, each node is assumed as a sensor. As previously mentioned, the sensors in M2M network can be classified into four groups, where some of them (groups 2 and 4) are mobile. Considering characteristics of different sensors, an energy consumption topology is required to be designed for prolonging system life and providing the needs of practical program in M2M network. Fig 2. Presents the steps of design phase for better eligibility using a flowchart.

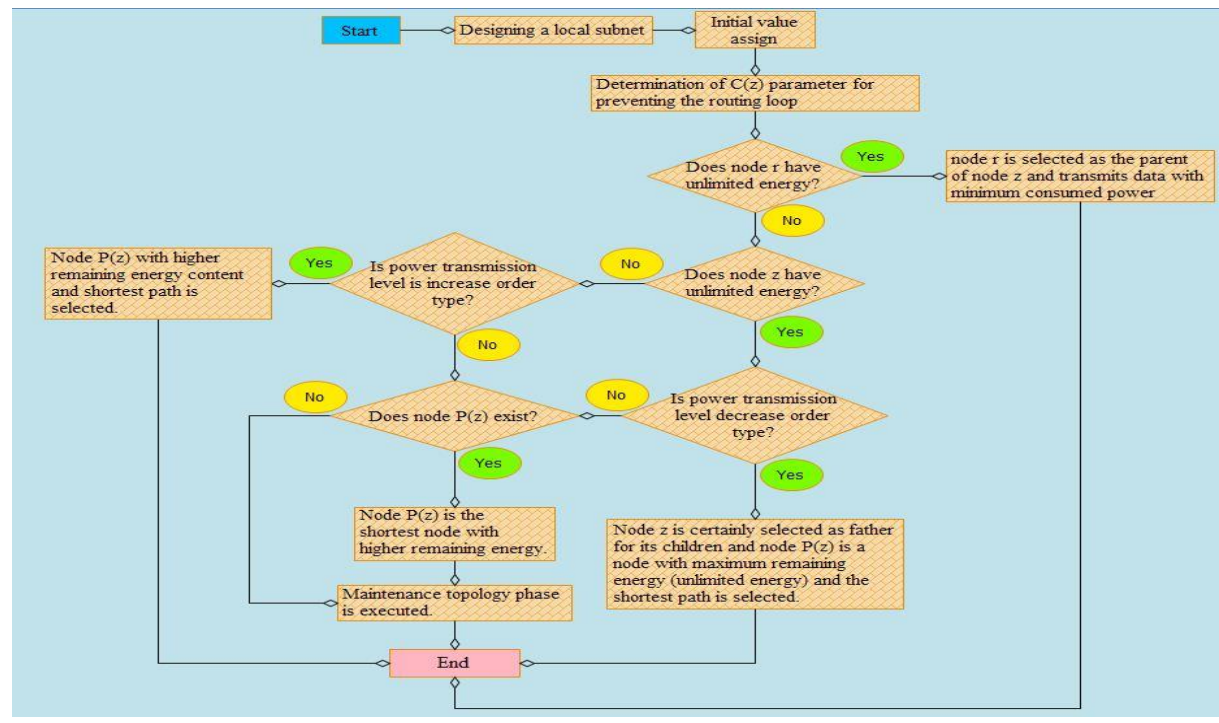

Fig. 2. Design phase flowchart.

\subsection{Steps of designing the proposed topology}

In the following lines, the algorithm for phase design is shown:

1- Let $\rho r m(i)$ be the remaining power of node $\mathrm{i}$

Let's be the Sink node

Let HC (i) be the hop count from node i to $s$ in the tree

2- Set $\mathrm{P}(\mathrm{s})==\varnothing$

3 - For every node $\mathrm{z} \neq \mathrm{s}$ : 
4- Set local graph of node z: Gz (N (z), L (z)) where $\mathrm{N}(\mathrm{z}) \in \mathrm{N}$ and $\mathrm{L}(\mathrm{z}) \in \mathrm{E}$

5- For all $x \in N(z)$ then

if $\mathrm{HC}(\mathrm{r}) \leq \mathrm{HC}(\mathrm{z})$

Set $\mathrm{P}(\mathrm{z})=\mathrm{r}$

\# Parent Selection by Energy remaining criteria

6- If $\operatorname{prm}(\mathrm{r})==\infty$ and no-loop $(\mathrm{z}, \mathrm{r})==0$ then

7- Set $P(z)=r$

\# Node $\mathrm{z}$ send data to $\mathrm{P}(\mathrm{z})$ with minimum transmission power

8- Set $\rho t x(z)=\rho \min (P(z), z)$

9- Else

\# determines power level for increase and decrease order

If $\operatorname{\rho rm}(\mathrm{z})==\infty$ then

10 - If $\rho p l(z)$ in decrease order and

11- If $\mathrm{HC}(\mathrm{r}) \leq \mathrm{HC}(\mathrm{z})$ then

Set $\mathrm{P}(\mathrm{z})=\operatorname{Max}\{\operatorname{\rho rm}(\mathrm{r})\}$

12- Set $\rho \operatorname{tx}(\mathrm{z})=\rho \min (\mathrm{P}(\mathrm{z}), \mathrm{z})$

13- End if

14- Else

15- If $\rho \operatorname{tx}(\mathrm{z})$ in increase order

16- If $\mathrm{HC}(\mathrm{r}) \leq \mathrm{HC}(\mathrm{z})$ then

17- Set $\mathrm{P}(\mathrm{z})=\operatorname{argmax}\{\rho \operatorname{rrm}(\mathrm{r})\}$

Set $\rho \operatorname{tx}(\mathrm{z})=\rho \min (\mathrm{P}(\mathrm{z}), \mathrm{z})$

18- End if

19- End If

20- End if

21- If $\mathrm{P}(\mathrm{z})=\varnothing$ then

22- Set $\mathrm{P}(\mathrm{z})=\operatorname{Max}\{\operatorname{\rho rm}(\mathrm{r})\}$

23- End If

24-Trigger topology maintenance Algorithm

Lines 1 to 4 of this algorithm indicate initial value assignment, where each sensor node creates a local graph. In line 5, to prevent routing loop, the hop number smaller or equal than sink node is used. In this way, selection of parent node depends on node number less than sink node. In lines 6 to 8, node of z sensor takes a neighbor node with unlimited energy supply as parent node. After selecting the parent nodes, the nodes can transmit data with minimum transmission power. In lines 9-12, if sensor node $\mathrm{z}$ has unlimited energy source, the transmission lever is decrease order. In this state, the sensor node can transmit data packets with maximum consumption power using an unlimited energy supply. In line 15, if sensor node $\mathrm{z}$ has limited (battery) supply source, transmission power takes the increase order form. In lines 16 and 17, for transmission power level, node $\mathrm{z}$ finds node $\mathrm{r}$ with maximum remaining energy and shortest path. In lines 21 and 22 , if there is no parent node, then the node with shortest path to sink node and maximum remaining energy would be assigned as the parent node. In Line 24, topology maintenance process is run. Then, the number of hops up to sink node (hop count, HC) is determined for each sensor node. Next, using the field based routing method, sink node distributes hop counts in the network and the nodes selected the shortest path based on the hop counts up to sink node [13]. 
The main purpose of this process is to design and develop a reliable topology with low energy consumption. Therefore, if node $\mathrm{r}$ close to node $\mathrm{z}$ has maximum remaining energy, node $\mathrm{z}$ can select node $\mathrm{r}$ as parent if it has minimum hop count up to sink node and a secure path in terms of energy level. In other words, during selection of parent node, the first priority is with less hop count up to sink node, and the second priority is the maximum remaining energy up of the path up to sink node. Here, the remaining energy of the path is energy of the nodes with transfer transmission traffic up to sink node. Moreover, it is not required to adjust the entire topology, and modifying the part related to the given node suffices.

\subsection{Maintenance phase of the proposed topology}

The main goal of topology design is to produce a topology for energy consumption management. Maintenance topology is defined as a process which alternatively restores and reconstructs the topology of the present network whenever the efficiency of the present topology is dropped. Therefore, the mechanism of maintenance topology results in creation of a new topology for energy consumption management of the sensor nodes and prolonging the network life. According the previous works, it can be stated that in a wireless sensor network, the data traffic load is not constantly distributed on the nodes in a unified mode. For example, sensors which have one node distance from sink node, carry a heavy load which reduces network life and efficiency in long run. This imbalance between the nodes' energy develops bottleneck effect around the sink node $[15,16]$. Which is among the most important problems for life length of wireless sensor networks. To analyse this problem one can use the results of pervious works, as well as applying dynamic "on the fly" topology maintenance techniques [14]. The dynamic topology mechanisms involve many advantages, as they are used for energy consumption optimization topology in sensor networks.

\subsection{Steps for running the proposed maintenance topology}

Fig 3. Presents the steps of maintenance topology for better eligibility in the form of a flowchart.

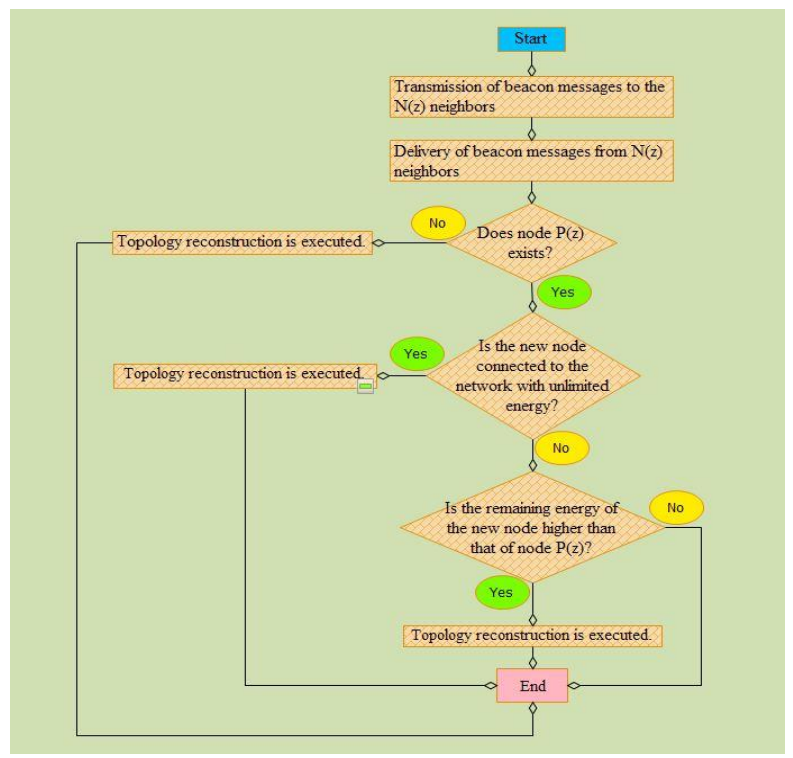

Fig. 3. Flowchart of topology maintenance phase. 
In the following lines, the algorithm of maintenance phase is presented:

1- Broadcast beacon message to neighbor $\mathrm{N}(\mathrm{z})$

2- Receive beacon message from each neighbor $\mathrm{N}(\mathrm{z})$

3- If $\mathrm{P}(\mathrm{z})$ is lost then

4- Trigger Topology Reconstruction Algorithm

5- End If

6- If $\operatorname{\rho rm}(\mathrm{A}(\mathrm{z}))=\infty$ then

7- Trigger Topology Reconstruction Algorithm

8- End If

9- If $\operatorname{\rho rm}(\mathrm{P}(\mathrm{z}))<\operatorname{\rho rm}(\mathrm{A}(\mathrm{z}))$ then

10- Trigger Topology Reconstruction Algorithm

11- End If

Lines 1 and 2 of this topology indicate that node $\mathrm{z}$ broadcasts beacon message to its neighboring node, $\mathrm{N}(\mathrm{z})$ during the maintenance topology phase. Similarly, each sensor node can deliver beacon message from its neighbor. The beacon packets transmitted to the neighboring nodes contain the following information:

Src: is used for identification of each packet;

Energy state: indicates remaining energy of each node;

Parent node: is an index which shows whether the node is linked to sink or not;

Hop count: is the number of wireless links in path from sink node to the given node; and

PS (power supply) state: which represents power supply of each node and whether the supply is limited or unlimited.

In line 3, each node can gather the information of neighbors through the beacon packets. In line 4-12, each node uses three decision making rules for making new topology structure:

1. If parent node of a sensor is deactivated, the given sensor node must select another parent based on the mentioned procedure.

2. If a sensor node with unlimited energy is linked to the network, it should introduce itself as a node with unlimited node, so that other nodes can select it as parent, on one hand, and this parent node is linked to sink node or other sensor node with unlimited energy supply, on the other hand.

If $\mathrm{z}$ sensor node is linked to a sensor node with unlimited energy source, there is no need for parent change unless the parent node is not deactivated. But if $\mathrm{z}$ sensor node is linked to a node with limited energy supply, once the remaining energy of parent node is less than that of alternative node, A (z), parent node should be changed. Thus, the alternative node selects a sensor node with unlimited energy or with higher remaining energy as the parent sensor node. Through the maintenance topology process, each sensor node makes topology using one of these three rules.

\section{Simulation Results}

In this section, effects and results of the proposed algorithm are presented and compared. Simulations were carried out using the NS-2 software. Table 1 presents simulation parameters. 
Table 1. Values of Parameters for simulation

\begin{tabular}{cc} 
Simulator & NS2 2.34 \\
AOding protocol & AOV \\
Simulation (Sec) & $1000 \mathrm{~m} \mathrm{X} 1000 \mathrm{~m}$ \\
Simulation area & 100 \\
Number of nodes & $10 \mathrm{~m}$ \\
Transmission range & 512 bytes/packet \\
Data payload & 100 \\
Queue Size & 4 packets/sec \\
Packet rate & CBR (UDP) \\
Traffic type & \\
\hline
\end{tabular}

Fig 4. Presents network life length measurements using or remaining energy of the network from the moment that first sensor triggers transmission process. As expected, the proposed algorithm has higher remaining energy as compared to the uncontrolled method.

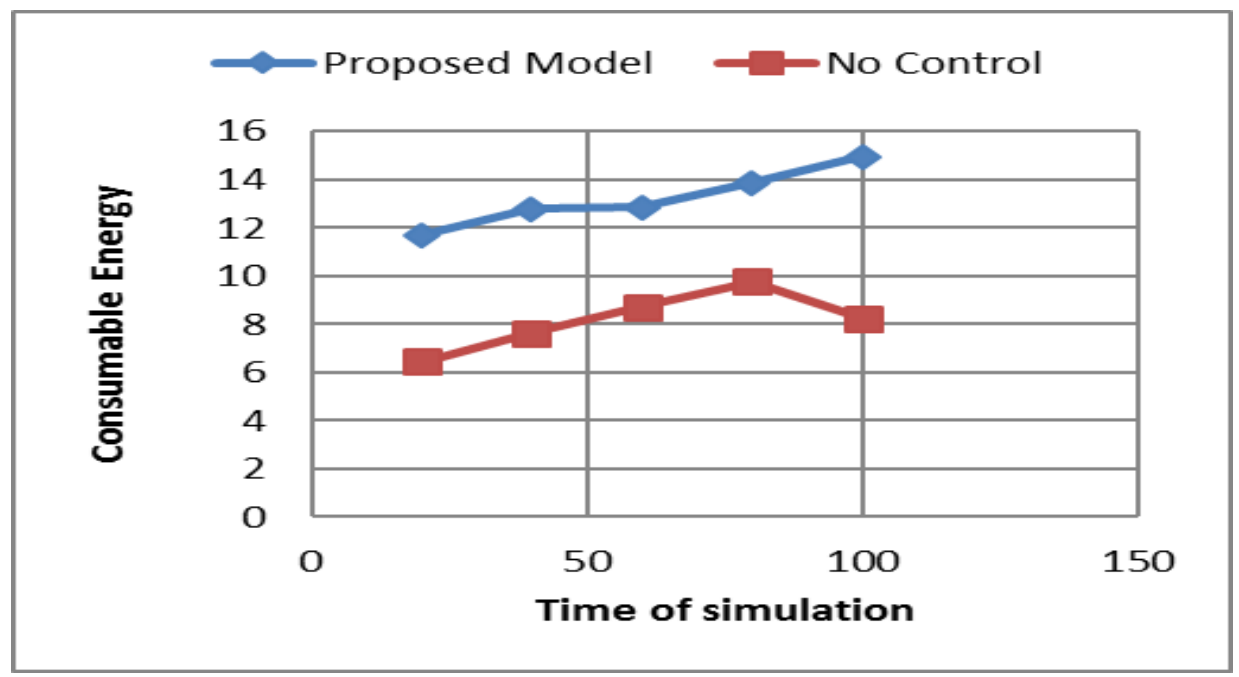

Fig. 4. Consumable energy

Fig 5. Illustrates the proposed algorithm in terms of packet delivery ratio (PDR) of healthy packets for different times. 


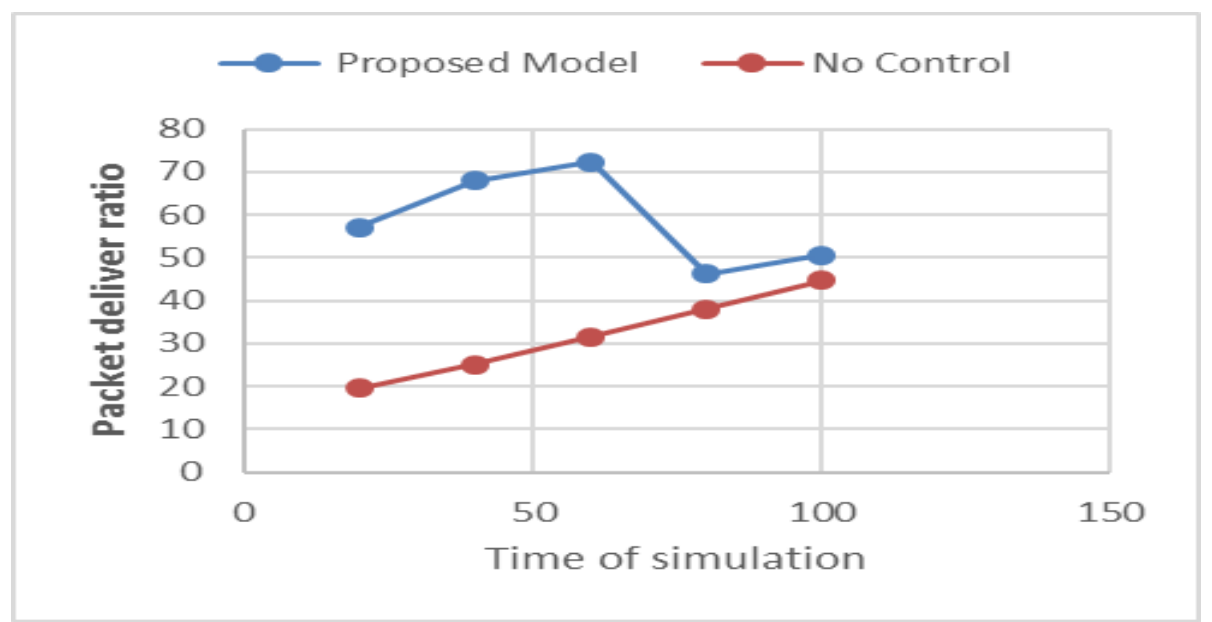

Fig. 5. Packet delivery ratio.

As shown in the Fig 5., PDR is higher for specific times. Fig 6. Presents throughput of the network.

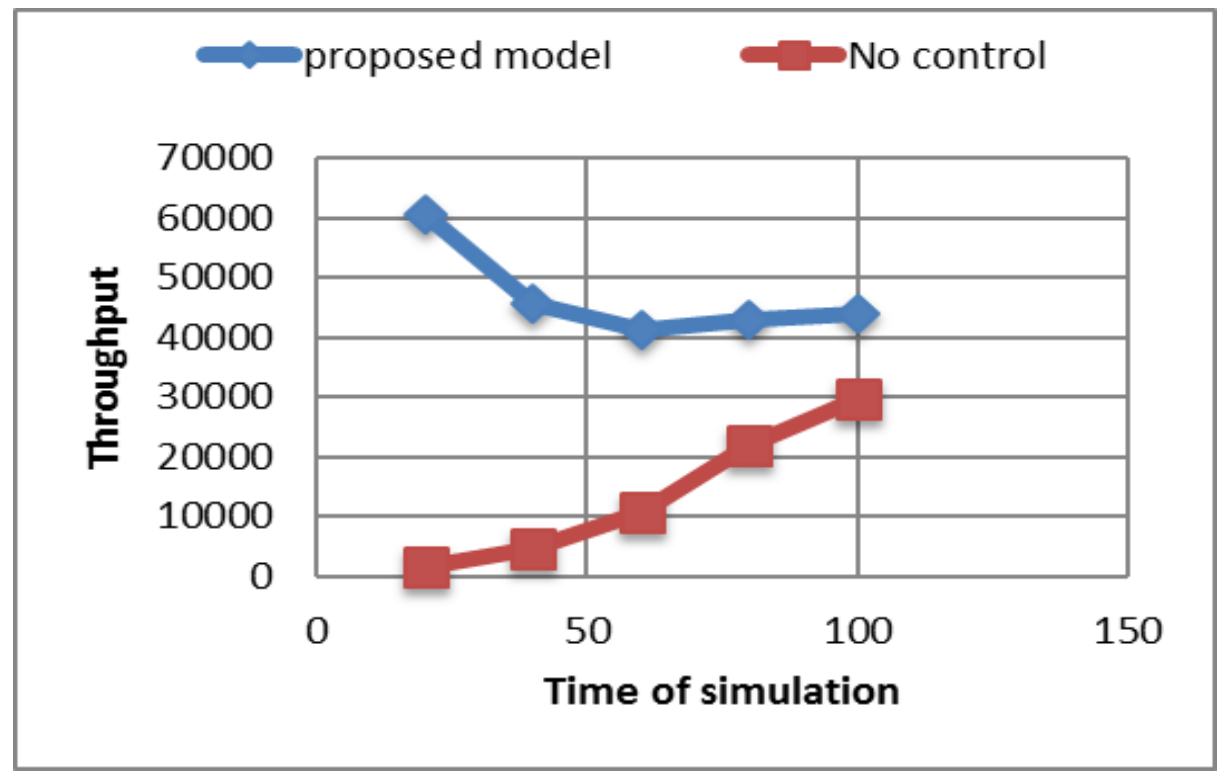

Fig. 6. Throughput.

The proposed algorithm indicates a very good throughput at the beginning stages, but at time proceeds the throughput of the network is lowered. However, the network indicates an acceptable overall performance.

As shown in Fig 7. Normalized routing load involves a better performance. 


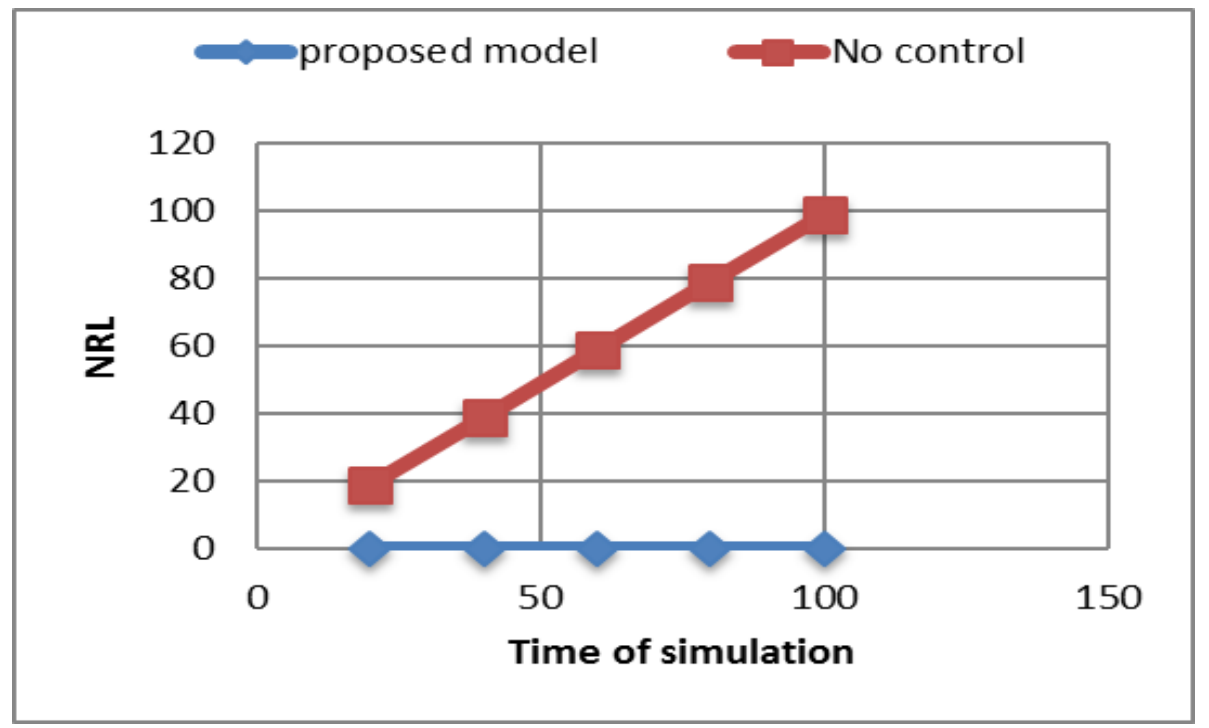

Fig. 7. Normalized routing load.

Considering the strict algorithms about using the hop in proposed algorithm, Fig 8. Unexpectedly shows that the applied hops in the proposed method have better behavior as compared to the uncontrolled method.

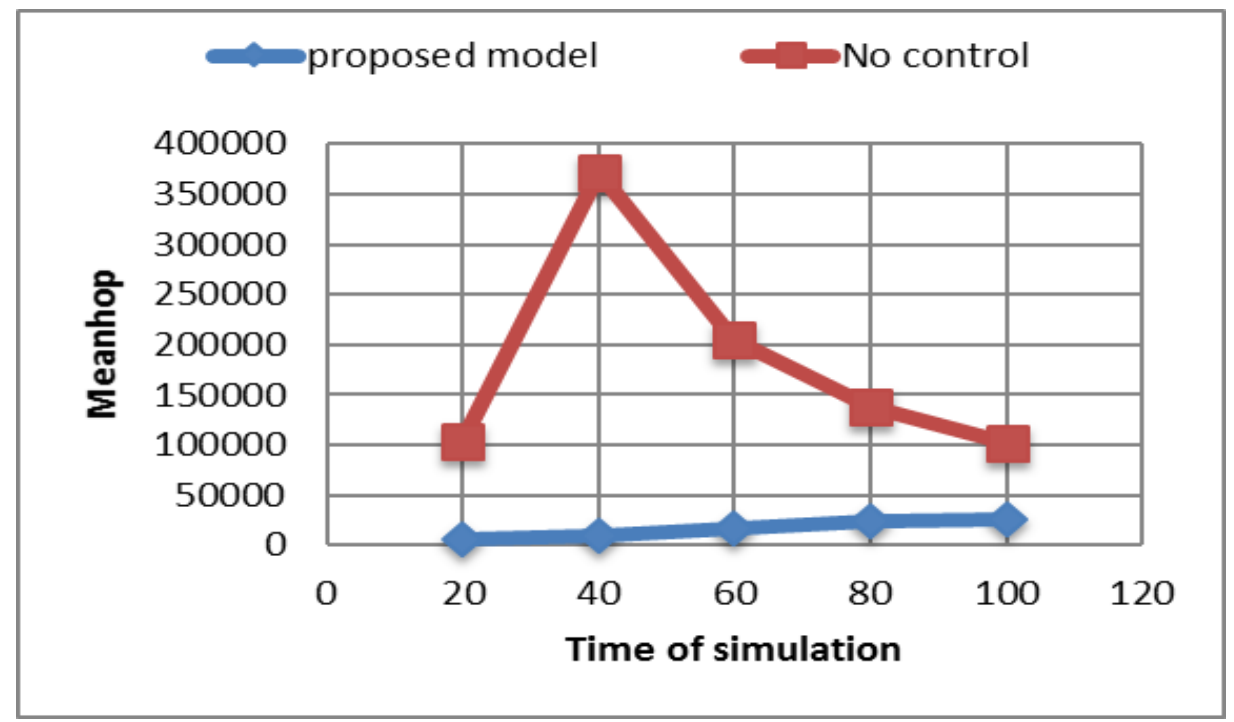

Fig. 8. Mean hop.

Fig 9. Indicates that at the simulation time increases, packet loss does not significantly change and a continuously acceptable behaviour is observed. 


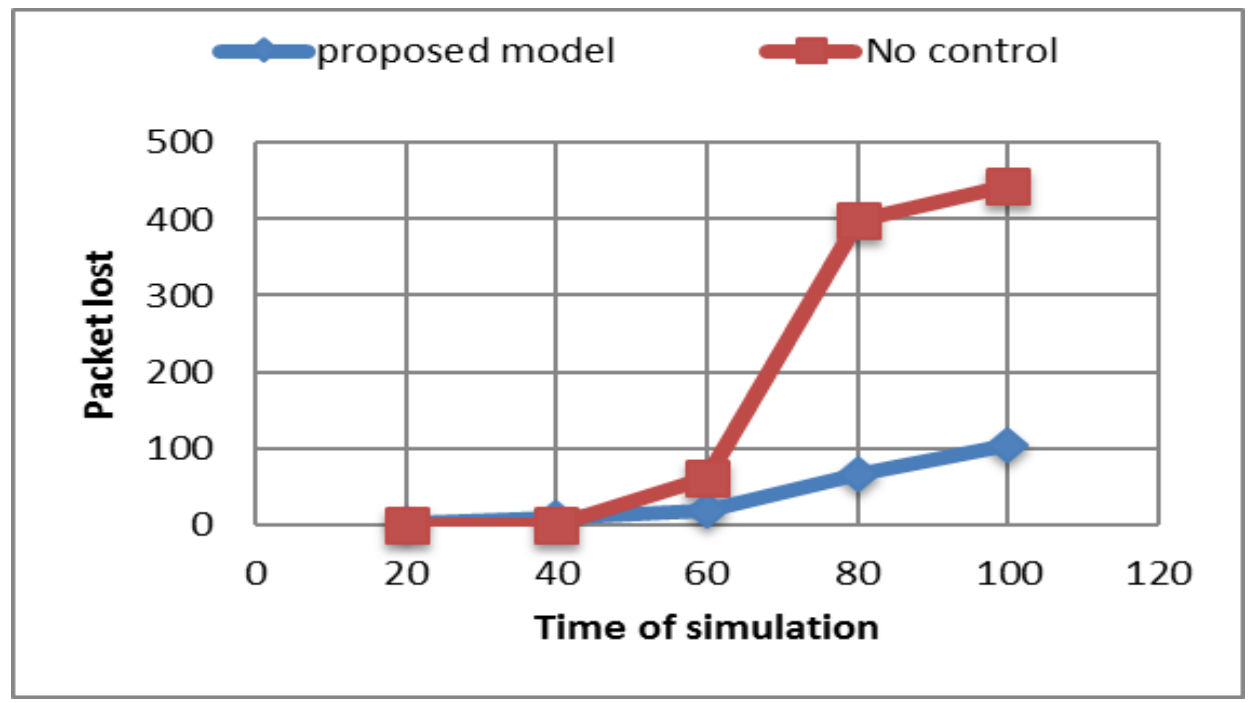

Fig. 9. Packet loss

\section{Conclusion}

Machine to Machine communication (M2M) is a new technology for communication between network-based equipment without human involvement. As implied by the performance of this communications, a large number of machines with various performances would be connected to this network in the few coming years [15], introducing an important challenge for expansibility of M2M networks. One of the agents playing a key role in these communications is wireless sensor networks (WSN) [16]. In this research some investigations were conducted for improving the expansibility of M2M networks through the topology control of WSNs and it was observed that the previous works are majorly focus on design topology. However, the topology maintenance should be also considered during the topology control design. Therefore, in this work an algorithm was proposed for prolonging system life though the energy consumption management, which focuses on both topology design and maintenance phases. The simulation results prove that the proposed algorithm not only prolong network life, but also effectively enhance network service quality.

\section{Reference}

[1] Akbar MAJIDI, Hamid MIRVAZIRI. BDCC: Backpressure routing and dynamic prioritization for congestion control in WMSNs. I.J. Computer Network and Information Security; 2014, 5, 29-34.

[2] Akbar MAJIDI, Hamid MIRVAZIRI. Congestion Control in WMSNs by Reducing Congestion and Free Resources to Set Accurate Rates and Priority. I.J. Computer Network and Information Security; 2014, 9, $37-42$.

[3] N. Tekbiyik and E. Uysal-Biyikoglu, "Energy efficient wireless unicast routing alternatives for machineto-machine networks," Journal of Network and Computer Applications, vol. 34, no. 5, pp. 1587-1614, 2011.

[4] Z. M. Fadlullah, M. M. Fouda, N. Kato, A. Takeuchi, N. Iwasaki, and Y. Nozaki, "Toward intelligent machine-to-machine communications in smart grid," IEEE Communications Magazine, vol. 49, no. 4, pp. 60-65, 2011. 
[5] D. Niyato, L. Xiao, and P. Wang, "Machine-to-machine communications for home energy management system in smart grid," IEEE Communications Magazine, vol. 49, no. 4, pp. 53-59, 2011.

[6] H. Sethu and T. Gerety, "A new distributed topology control algorithm for wireless environments with non-uniform path loss and multipath propagation," Ad Hoc Networks, vol. 8, no. 3, pp. 280-294, 2010.

[7] S. Rizvi, H. K. Qureshi, S. A. Khayam, V. Rakocevic b, and M. Rajarajan, "A1: an energy efficient topology control algorithm for connected area coverage in wireless sensor networks," Journal of Network and Computer Applications, vol. 35, no. 2, pp. 597-605, 2012.

[8] T. M. Chiwewe and G. P. Hancke, "A distributed topology control technique for low interference and energy efficiency in wireless sensor networks," IEEE Transactions on Industrial Informatics, vol. 8, no. 1, pp. 11-19, 2012.

[9] H. " Uster and H. Lin, "Integrated topology control and routing in wireless sensor networks for prolonged network lifetime," Ad Hoc Networks, vol. 9, no. 5, pp. 835-851, 2011.

[10] J. Ma, M. Gao, Q. Zhang, and L. M. Ni, "Energy-efficient localized topology control algorithms in IEEE 802.15.4- based sensor networks," IEEE Transactions on Parallel and Distributed Systems, vol. 18, no. 5, pp. 711-720, 2007.

[11] David Boswarthick, Omar Elloumi, and Olivier Hersent "M2M communications: A systems approach "ISBN 978-1-119-99475-6, 2012, Willy, 1th Edition.

[12] IEEE Standard 802. 15.4,"Wireless Medium Access Control (MAC) and Physical Layer (PHY) Specifications for Low-Rate Wireless Personal Area Networks (LR-WPANs)", 2006.

[13] Malaz K, Dujeong L, June-Koo Kevin R, "Distributed Potential Field Based Routing and Autonomous Load Balancing for Wireless Mesh Networks" IEEE COMMUNICATIONS LETTERS, VOL. 13, NO. 6, JUNE 2009.

[14] M. A. Labrador and P. M. Wightman, “Topology Control in Wireless Sensor Networks with a Companion Simulation Tool for Teaching and Research" Springer, Berlin, Germany, 2009.

[15] Olliver Hersent, David Boswarthick, Omar Elloumi, "The Internet of Things: Key Applications and Protocols" ISBN-13: 978-1119994350, February 6, 2012, Edition: 2.

[16] Min Chen, Jiafu Wan, Fang Li "Machine-to-Machine Communications: Architectures, Standards and Applications" KSII TRANSACTIONS ON INTERNET AND INFORMATION SYSTEMS VOL. 6, NO. 2, February 28, 2012.

\section{Authors' Profiles}

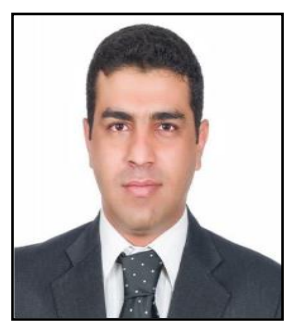

Mohammad Amini was born on September 1982 in Dena-Sisakht, Iran. He received the B.Sc. degree in Electrical Engineering majoring in Electronics in 2007 and the M.Sc. degree in Information Technology majoring in Computer Networks from Amirkabir University of Technology (Tehran Polytechnic), Tehran, Iran, in 2013. His current research interests are in the field of Computer Networks, Machine to Machine Communications (M2M), Wireless Sensor Networks (WSN), Future Internet and ICT 


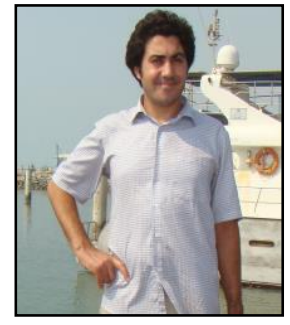

Intrusion Detection Protocol (VoIP).

Akbar Majidi: was born on January 1986 in Ardabil- Germi, Iran. He received his B.Sc. degree in Software Engineering in 2009, and M.Sc. degree in Architecture of Computer Systems from Iran University of Science and Research Branch, Kerman, Iran, in 2013. $\mathrm{He}$ has been the founder and also the president of Networks Laboratory and Advance Programming (NLAP) in Ardebil-Germi, Iran. His research interests are Information Centric Networking (ICN), Software-defined networking (SDN), Wireless Sensor and Wireless Multimedia Sensor Networks (WMSNs), Graph Theory and Ring Theory, Adhoc Network Protocols and Architectures, Congestion Control and Buffer Management, System and Attacks, Radio Frequency Identification (RFID) andVoice-over Internet

How to cite this paper: Mohammad Amini, Akbar Majidi,"Introducing a Novel Algorithm for Energy Consumption Management for M2M Network Expandability through Topology Control within Nodes", IJWMT, vol.4, no.4, pp.59-71, 2014.DOI: 10.5815/ijwmt.2014.04.05 